\title{
A New Mathematical Formulation for a Phase Change Problem with a Memory Flux
}

\author{
Sabrina D. Roscani, Julieta Bollati, and Domingo A. Tarzia \\ CONICET - Depto. Matemática, FCE, Univ. Austral, \\ Paraguay 1950, S2000FZF Rosario, Argentina \\ (sabrinaroscani@gmail.com, jbollati@austral.edu.ar, dtarzia@austral.edu.ar)
}

\begin{abstract}
:
A mathematical formulation for a one-phase change problem in a form of Stefan problem with a memory flux is obtained. The hypothesis that the integral of weighted backward fluxes is proportional to the gradient of the temperature is considered. The model that arises involves fractional derivatives with respect to time both in the sense of Caputo and of Riemann-Liouville. An integral relation for the free boundary, which is equivalent to the "fractional Stefan condition", is also obtained.

Keywords: Stefan problem, Fractional diffusion equation, Riemann-Liouville derivative, Caputo derivative, memory flux, equivalent integral relation.
\end{abstract}

AMS: Primary: 35R35, 26A33, 35C05. Secondary: 33E20, 80A22.

Note: This paper is now published (in revised form) in Chaos, Solitons and Fractals 116 (2018), p.p. 340-347, DOI:10.1016/j.chaos.2018.09.023, and is available online at www.elsevier.com/locate/chaos, so always cite it with the journal's coordinates.

\section{Introduction}

The theory related to heat diffusion has been extensively developed in the last century. Modelling classical heat diffusion comes hand in hand with Fourier Law. Nevertheless, we shall not forget that this famous law is an experimental phenomenological principle.

In the past 40 years, many generalized flux models of the classical one (i.e. the one derived from Fourier Law) were proposed in the literature and accepted by the scientific community. See e.g. [8, 16 18, 37].

In this paper a phase change problem for heat diffusion under the hypothesis that the heat flux is a flux with memory is analysed. This kind of problems are known in the literature as Stefan problems [41,42].

The model obtained under the memory assumption is known as an anomalous diffusion model, and it is governed by fractional diffusion equations. There is a vast literature in the subject of fractional diffusion equations. We refer the reader to [24, 30, 31] and references therein. 
The study of anomalous diffusion has its origins in the investigation of non-Brownian motions (Random walks). In that context it was observed that "the mean square displacement" of the particles is proportional to a power of the time, instead of being proportional just to time. An exhaustive work in this direction has been done by Metzler and Klafter [26]. Other articles in this direction are [19, 25, 27, 28]. It is worth mentioning that many works (see e.g. [3, 12, 40]) suggest that the anomalous diffusion is caused by heterogeneities in the domain.

Before presenting the problem, let us establish some usual notation related to heat conduction with the corresponding physical dimensions. Let us write $\mathbf{T}$ for temperature, $\mathbf{t}$ for time, $\mathbf{m}$ for mass and $\mathbf{X}$ for position.

$\begin{array}{ccc}u & \text { temperature } & {[\mathbf{T}]} \\ k & \text { thermal conductivity } & {\left[\frac{\mathbf{m} \mathbf{X}}{\mathbf{T t}^{3}}\right]} \\ \rho & \text { mass density } & {\left[\frac{\mathbf{m}}{\mathbf{X}^{3}}\right]} \\ c & \text { specific heat } & {\left[\frac{\mathbf{X}^{2}}{\mathbf{T t}^{2}}\right]} \\ d=\frac{k}{\rho c} & \text { diffusion coefficient } & {\left[\frac{\mathbf{X}^{2}}{\mathbf{t}}\right.} \\ l & \text { latent heat per unit mass } & {\left[\frac{\mathbf{X}^{2}}{\mathbf{t}^{2}}\right]}\end{array}$

Consider a temperature function $u=u(x, t)$ and its corresponding flux $J(x, t)$, both defined for a semi-infinite unidimensional material. From the First Principle of Thermodynamics, we deduce the continuity equation

$$
\rho c \frac{\partial u}{\partial t}(x, t)=-\frac{\partial J}{\partial x}(x, t)
$$

The aim of this work is to derive a model by considering a special non-local memory flux. For example, Gurtin and Pipkin [15] (experts in continuum mechanics and heat transfer) proposed in 1968 a general theory of heat conduction with finite velocity waves through the following non local flux law:

$$
J(x, t)=K(t) *\left(-k \frac{\partial}{\partial x} u(x, t)\right)=-k \int_{-\infty}^{t} K(t-\tau) \frac{\partial}{\partial x} u(x, \tau) \mathrm{d} \tau,
$$

where $K$ is a positive decreasing kernel which verifies $K(s) \rightarrow 0$ when $s \rightarrow \infty$.

Let us comment on some different explicit and implicit definitions of fluxes, and their effects on the resulting governing equations:

- Explicit forms for the flux: $J(x, t)=F(x, t)$

The classical law for the flux is the Fourier Law, which states that the flux $\mathrm{J}$ is proportional to the temperature gradient, that is:

$$
J(x, t)=-k \frac{\partial}{\partial x} u(x, t) .
$$


If alternatively suppose that the flux at the point $(x, t)$ is proportional to the total flux, then the given law is the following

$$
J(x, t)=\frac{1}{\tilde{\tau}} \int_{-\infty}^{t}-k \frac{\partial}{\partial x} u(x, \tau) \mathrm{d} \tau .
$$

In (5), $\tilde{\tau}$ is a constant whose physical dimension is time. Another interesting thing is that (5) can be interpreted as a generalized sum of backward fluxes, where every local flux has the same "relevance".

The following expression for the flux is a generalized sum of weighted backward fluxes. There is now a kernel which assigns more weight ("importance") to the nearest temperature gradients, that is:

$$
J(x, t)=-\frac{\eta_{\alpha}}{\Gamma(\alpha)} \int_{-\infty}^{t}(t-\tau)^{\alpha-1} k \frac{\partial}{\partial x} u(x, \tau) \mathrm{d} \tau .
$$

Here, $\alpha$ is a constant in the interval $(0,1)$ that plays an important role, and $\eta_{\alpha}$ is a constant imposed to equate units of measures. Both will be specified later.

Note that (4) and (6) result from considering the kernels $K_{1}(t) \equiv \delta(t)$ and $K_{2}(t)=\eta_{\alpha} \frac{t^{\alpha-1}}{\Gamma(\alpha)}$, respectively, in the generalized flux equation (3).

- Implicit forms for the flux: $F(x, t, J(x, t))=G(x, t)$.

One of the most famous formulations for the flux, is given by the Cattaneo's equation [6]

$$
J(x, t)+\tilde{\tau} \frac{\partial}{\partial t} J(x, t)=-k \frac{\partial}{\partial x} u(x, t),
$$

which was proposed with the aim of introducing an alternative to the "unphysical" property of the diffusion equation known as infinite speed of propagation. Equation (7) can be seen as a first order Taylor approximation of (8) in which the flux is allowed to adjust to the gradient of the temperature according to a relaxation time $\tilde{\tau}$,

$$
J(x, t+\tilde{\tau})=-k \frac{\partial}{\partial x} u(x, t)
$$

Another approach assumes that the integral of the back fluxes, at the current time, is proportional to the gradient of the temperature:

$$
\frac{1}{\tilde{\tau}} \int_{-\infty}^{t} J(x, \tau) \mathrm{d} \tau=-k \frac{\partial}{\partial x} u(x, \tau) .
$$


Yet another formulation considers that the integral of the weighted backward fluxes at the current time, is proportional to the gradient of the temperature:

$$
\frac{\nu_{\alpha}}{\Gamma(1-\alpha)} \int_{-\infty}^{t}(t-\tau)^{-\alpha} J(x, \tau) \mathrm{d} \tau=-k \frac{\partial}{\partial x} u(x, \tau) .
$$

Note 1. Although when we talk about backward fluxes it is logical to consider the lower limit of the integral at $-\infty$, we can suppose that the function $u$ has remained constant (for some reason) for all $t<0$, where with 0 we refer to a certain initial time. Moreover, under this condition, that is $u(x, t) \equiv u_{0}$, for every $t<0$, the expressions (6) and (9) become

$$
J(x, t)=-\frac{\eta_{\alpha}}{\Gamma(\alpha)} \int_{0}^{t}(t-\tau)^{\alpha-1} k \frac{\partial}{\partial x} u(x, \tau) \mathrm{d} \tau,
$$

and

$$
\frac{\nu_{\alpha}}{\Gamma(1-\alpha)} \int_{0}^{t}(t-\tau)^{-\alpha} J(x, \tau) \mathrm{d} \tau=-k \frac{\partial}{\partial x} u(x, \tau)
$$

respectively.

Expressions (10), (11) are closely linked to fractional calculus. Let us present the basic definitions that will be employed throughout the article.

Definition 1. Let $[a, b] \subset \mathbb{R}$ and $\alpha \in \mathbb{R}^{+}$be such that $n-1<\alpha \leq n$.

1. For $f \in L^{1}[a, b]$, we define the fractional Riemann-Liouville integral of order $\alpha$ as

$$
{ }_{a} I^{\alpha} f(t)=\frac{1}{\Gamma(\alpha)} \int_{a}^{t}(t-\tau)^{\alpha-1} f(\tau) \mathrm{d} \tau .
$$

2. For $f \in A C^{n}[a, b]=\left\{f \mid f^{(n-1)}\right.$ is absolutely continuous on $\left.[a, b]\right\}$, we define the fractional Riemann-Liouville derivative of order $\alpha$ as

$$
{ }_{a}^{R L} D^{\alpha} f(t)=\left[D_{a}^{n} I^{n-\alpha} f\right](t)=\frac{1}{\Gamma(n-\alpha)} \frac{d^{n}}{d t^{n}} \int_{a}^{t}(t-\tau)^{n-\alpha-1} f(\tau) \mathrm{d} \tau .
$$

3. For $f \in W^{n}(a, b)=\left\{f \mid f^{(n)} \in L^{1}[a, b]\right\}$, we define the fractional Caputo derivative of order $\alpha$ as

$$
{ }_{a}^{C} D^{\alpha} f(t)=\left[{ }_{a} I^{n-\alpha}\left(D^{n} f\right)\right](t)=\left\{\begin{array}{lc}
\frac{1}{\Gamma(n-\alpha)} \int_{a}^{t}(t-\tau)^{n-\alpha-1} f^{(n)}(\tau) \mathrm{d} \tau, & n-1<\alpha<n, \\
f^{(n)}(t), & \alpha=n .
\end{array}\right.
$$


Note 2. With these definitions, equations (10) and (11) can be rewritten as

$$
J(x, t)=-\eta_{\alpha} k_{0} I_{t}^{\alpha} \frac{\partial}{\partial x} u(x, t)
$$

and

$$
\nu_{\alpha 0} I_{t}^{1-\alpha} J(x, \tau)=-k \frac{\partial}{\partial x} u(x, t) .
$$

Table 1 exhibits the governing equations derived from (2) for the different choices of the flux $J$.

Table 1: Flux and diffusion equations

\begin{tabular}{|l|l|l|}
\hline Equation for the Flux & Resulting Diffusion Eq. & Observations \\
\hline$J=-k \frac{\partial u}{\partial x}$ & $\frac{\partial u}{\partial t}=d \frac{\partial^{2} u}{\partial x^{2}}$ & Heat equation \\
\hline$J=-\frac{1}{\tilde{\tau}} \int_{0}^{t} k \frac{\partial u}{\partial x} d \tau$ & $\frac{\partial^{2} u}{\partial t^{2}}=\frac{d}{\tilde{\tau}} \frac{\partial^{2} u}{\partial x^{2}}$ & Wave equation \\
\hline$J=-k \eta_{\alpha} I_{t}^{\alpha} \frac{\partial u}{\partial x}$ & ${ }_{0}^{C} D_{t}^{1+\alpha} u=\eta_{\alpha} d \frac{\partial^{2} u}{\partial x^{2}}$ & Superdiffusion equation \\
\hline$J+\tilde{\tau} \frac{\partial}{\partial t} J=-k \frac{\partial u}{\partial x}$ & $\frac{\partial u}{\partial t}+\tilde{\tau} \frac{\partial^{2} u}{\partial t^{2}}=\frac{\partial^{2} u}{\partial x^{2}}$ & Telegraph equation \\
\hline$\frac{1}{\tau^{*}} \int_{0}^{t} J \mathrm{~d} \tau=-k \frac{\partial u}{\partial x}$ & $\frac{\partial}{\partial t}\left(u-\frac{d}{\tau^{*}} \frac{\partial^{2} u}{\partial x^{2}}\right)=0$ & $\begin{array}{l}\text { Elliptic equation } \\
\text { with parameter } t\end{array}$ \\
\hline$\nu_{\alpha 0} I_{t}^{1-\alpha} J=-k \frac{\partial u}{\partial x}$ & ${ }_{0}^{C} D^{\alpha} u(x, t)=\frac{d}{\nu_{\alpha}} \frac{\partial^{2}}{\partial x^{2}} u(x, t)$ & Subdiffusion equation \\
\hline
\end{tabular}

There are many references about these different fluxes and their corresponding governing equations [5,6, 10, 24, 30, 31. Specially, the subdiffusion equation is one of the most studied in the last 10 years: The Cauchy problem [11,14,23,29], initial and boundary value problems [13, 38], maximum principles [1,21,22, 32]. Nevertheless, fractional phase change problems have been very poorly studied [2,43]. Some of these articles propose a physical approach [4,7,44, 46] and others do a purely mathematical treatment [20, 33, 34].

The goal of this paper is to present a new mathematical model for a one phase change problem with a memory flux, which derives in a fractional free boundary problem, such that the governing equations of this model are consistent both mathematically and physically speaking. We will pay special attention to the interchange of limits and integrals, which is a sensitive issue when working with fractional derivatives (see [36]).

In Section 2, some properties of fractional calculus which will be useful later are provided. In Section 3, a mathematical formulation for an instantaneous phase-change problem for 
a material with memory is presented. In this model, an implicit equation for the flux involving fractional integrals is used.

Finally, in Section 4, an equivalent formulation is presented, which allows us to give an integral relation for the free boundary, which we consider important in future research on existence and uniqueness of solutions, or properties of the free boundary.

\section{Preliminaries of Fractional Calculus}

Proposition 1. [9] The following properties involving the fractional integrals and derivatives hold:

1. The fractional Riemann-Liouville derivative is a left inverse operator of the fractional Riemann-Liouville integral of the same order $\alpha \in \mathbb{R}^{+}$. If $f \in L^{1}[a, b]$, then

$$
{ }_{a}^{R L} D^{\alpha}{ }_{a} I^{\alpha} f(t)=f(t) \quad \text { a.e. }
$$

2. The fractional Riemann-Liouville integral is not, in general, a left inverse operator of the fractional derivative of Riemann-Liouville.

In particular, if $0<\alpha<1$, then ${ }_{a} I^{\alpha}\left({ }_{a}^{R L} D^{\alpha} f\right)(t)=f(t)-\frac{{ }_{a} I^{1-\alpha} f\left(a^{+}\right)}{\Gamma(\alpha)(t-a)^{1-\alpha}}$.

3. If there exists some $\phi \in L^{1}(a, b)$ such that $f={ }_{a} I^{\alpha} \phi$, then

$$
{ }_{a} I_{a}^{\alpha}{ }_{a} L D^{\alpha} f(t)=f(t) \quad \text { a.e. }
$$

4. If $n-1<\alpha \leq n$ and $f \in A C^{n}[a, b]$, then

$$
{ }_{a}^{R L} D^{\alpha} f(t)=\sum_{k=0}^{n-1} \frac{f^{(k)}(a)}{\Gamma(1+k-\alpha)}(t-a)^{k-\alpha}+{ }_{a}^{C} D^{\alpha} f(t) .
$$

In particular, for $0<\alpha<1$, we have

$$
{ }_{a}^{R L} D^{\alpha} f(t)=\frac{f(a)}{\Gamma(1-\alpha)}(t-a)^{-\alpha}+{ }_{a}^{C} D^{\alpha} f(t) .
$$

Proposition 2. [39] The following limits hold:

1. If we set ${ }_{a} I^{0}=I d$, the identity operator, then for every $f \in L^{1}[a, b]$,

$$
\lim _{\alpha \searrow 0}{ }_{a} I^{\alpha} f(t)={ }_{a} I^{0} f(t)=f(t) .
$$


2. For every $f \in C^{1}(a, b)$,

$$
\lim _{\alpha \nearrow 1}{ }_{a}^{C} D^{\alpha} f(t)=f^{\prime}(t) \quad \text { and } \quad \lim _{\alpha \searrow 1}{ }_{a}^{C} D^{\alpha} f(t)=f^{\prime}(t)-f^{\prime}\left(0^{+}\right), \quad \forall t \in[a, b] .
$$

3. For every $f \in A C^{1}[a, b]$,

$$
\lim _{\alpha \nearrow 1}{ }_{a}^{R L} D^{\alpha} f(t)=f^{\prime}(t) \quad \text { and } \quad \lim _{\alpha \searrow 1}{ }_{a}^{R L} D^{\alpha} f(t)=f^{\prime}(t), \quad \text { a.e. } t \in(a, b) .
$$

Remark 1. If we consider a function $f$ supported in $[0, \infty)$ and $\chi_{\alpha}$ is the locally integrable function defined by

$$
\chi_{\alpha}(t)= \begin{cases}\frac{t^{\alpha-1}}{\Gamma(\alpha)} & \text { if } t>0, \\ 0 & \text { if } t \leq 0,\end{cases}
$$

then we have the following properties for $0<\alpha<1$ :

$$
\begin{gathered}
{ }_{a} I^{\alpha} f(t)=\left(\chi_{\alpha} * f\right)(t), \\
{ }_{a}^{R L} D^{\alpha} f(t)=\frac{d}{d t}\left(\chi_{1-\alpha} * f\right)(t), \\
{ }_{a}^{C} D^{\alpha} f(t)=\left(\chi_{1-\alpha}\right) * \frac{d}{d t} f(t) .
\end{gathered}
$$

\section{Modelling a Phase Change Problem with a flux with memory: A fractional Stefan problem}

The aim of this section is to formulate mathematical models associated to a one-dimensional fractional phase change problem.

The classical phase change problems for the heat equation obtained by considering the Fourier Law for the flux are known in the mathematical literature as free boundary problems, and under certain conditions as Stefan problems.

The fundamental equations involved in Stefan problems are: the heat equation and the Stefan condition (derived from the connection between the velocity of the free boundary and the heat fluxes of the two temperatures corresponding to the different phases).

We will focus on deriving the fractional diffusion equation and (making an abuse of language) the "fractional Stefan condition".

Physical problem: Melting of a semi-infinite slab $(0 \leq x<\infty)$ of a material with memory, which is at the melt temperature $T_{m}$, by imposing a constant temperature $T_{0}>T_{m}$ on the fixed face $x=0$. All the thermophysical parameters are constants. 
Mathematical problem Let $u=u(x, t)$ be the temperature and let $J(x, t)$ be the memory flux of the material at position $x$ and time $t$. Let $x=s(t)$ be the function representing the (unknown) position of the free boundary at time $t$ such that $s(0)=0$. We will assume that $s$ is an increasing function and consequently, an invertible function.

The flux modelling the material with memory is considered under the assumption that the generalized sum of the weighted backward fluxes at the current time is proportional to the gradient of the temperature, that is

$$
\nu_{\alpha h(x)} I_{t}^{1-\alpha} J(x, t)=-k \frac{\partial u}{\partial x}(x, t),
$$

where the initial time in the fractional integral is given by the function $h$ which gives us the time when the phase change occurs. That is

$$
h(x)=s^{-1}(x) \quad(\text { i.e. } \quad x=s(t)) .
$$

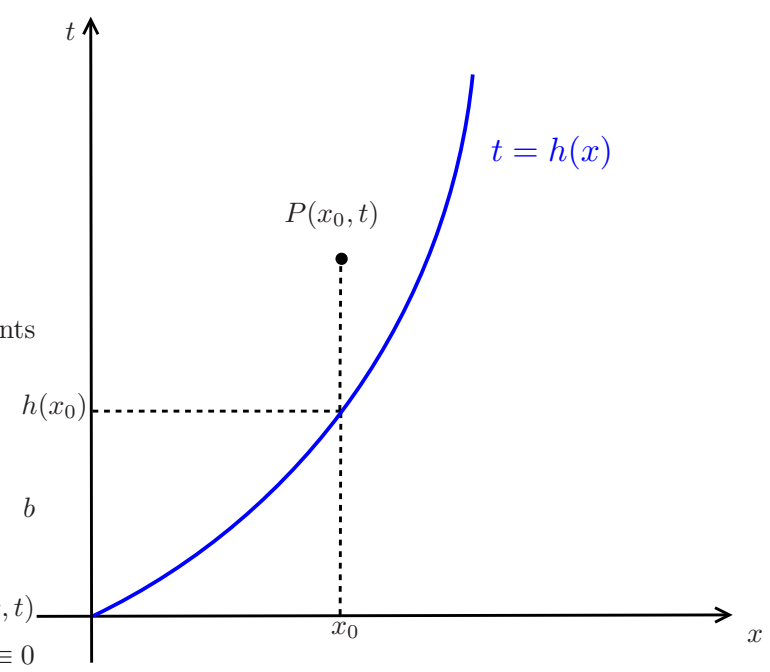

Figure 1: The free boundary $h(x)$ vs $x$.

The parameter $\nu_{\alpha}$ is a parameter with physical dimension such that

$$
\lim _{\alpha \nearrow 1} \nu_{\alpha}=1 .
$$

This parameter has been added to preserve the consistency with respect to the units of measure in equation (12). In fact, considering the units of measure given in (11), we have

$$
[J]=\left[k u_{x}\right]=\frac{\mathbf{m}}{\mathbf{t}^{3}},
$$




$$
\left[{ }_{h(x)} I_{t}^{1-\alpha} J(x, t)\right]=\left[\frac{1}{\Gamma(1-\alpha)} \int_{h(x)}^{t} \frac{J(x, \tau)}{(t-\tau)^{\alpha}} \mathrm{d} \tau\right]=\frac{\mathbf{m}}{\mathbf{t}^{3}} \frac{1}{\mathbf{t}^{\alpha}} \mathbf{t}=\frac{\mathbf{m}}{\mathbf{t}^{2+\alpha}} .
$$

Then, by (14), (15) and (12) one gets

$$
\left[\nu_{\alpha}\right]=\frac{\left[k \frac{\partial u}{\partial x}\right]}{\left.\left[h(x) I_{t}^{1-\alpha} J\right]\right)}=\frac{1}{\mathbf{t}^{1-\alpha}} .
$$

Remark 2. Due to the properties of the Riemann-Liouville integral, the limit expression for $\alpha=1$ in (12) yields the classical Fourier Law.

Remark 3. Notice that, since we are assuming that the temperature is constant for $x>s(t)$, then the gradient of the temperature is null in the region $x>s(t), t>0$, which implies that

$$
\nu_{\alpha} I_{t}^{1-\alpha} J(x, t)=0, \quad \forall x>s(t), t>0 .
$$

Applying the inverse operator ${ }_{0}^{R L} D_{t}^{1-\alpha}$ to both sides of equation (17) leads to

$$
\nu_{\alpha} J(x, t)={ }_{0}^{R L} D_{t}^{1-\alpha} 0=0, \quad \forall x>s(t), t>0 .
$$

Then, for every $(x, t)$ such that $0<x<s(t), t>0$ it results that

$$
\begin{aligned}
\nu_{\alpha 0} I_{t}^{1-\alpha} J(x, t) & =\frac{\nu_{\alpha}}{\Gamma(1-\alpha)} \int_{0}^{t} \frac{J(x, \tau)}{(t-\tau)^{\alpha}} \mathrm{d} \tau \\
& =\frac{\nu_{\alpha}}{\Gamma(1-\alpha)} \int_{0}^{h(x)} \frac{0}{(t-\tau)^{\alpha}} \mathrm{d} \tau+\frac{\nu_{\alpha}}{\Gamma(1-\alpha)} \int_{h(x)}^{t} \frac{J(x, \tau)}{(t-\tau)^{\alpha}} \mathrm{d} \tau \\
& =\nu_{\alpha h(x)} I_{t}^{1-\alpha} J(x, t) .
\end{aligned}
$$

So, assuming that $u(x, t) \equiv T_{m}$ in the region $x>s(t), t>0$, condition (12) is equivalent to

$$
\nu_{\alpha 0} I_{t}^{1-\alpha} J(x, t)=-k \frac{\partial u}{\partial x}(x, t) \quad \forall 0<x<s(t), t>0 .
$$

However, in the following, expression (12) will be chosen since the dependence on starting time (linked to the free boundary) may be overlooked if we consider (18).

Now, being the Riemann-Liouville fractional derivative of order $1-\alpha$ a left inverse operator of the fractional Riemann-Liouville integral (Proposition 1-11), an explicit expression for the memory flux at position $x$ and time $t$ can be derived, and it is given by

$$
J(x, t)=-\frac{k}{\nu_{\alpha}} \underset{h L}{R L} D_{t}^{1-\alpha} \frac{\partial u}{\partial x}(x, t),
$$

or

$$
J(x, t)=-\frac{k}{\nu_{\alpha}} \frac{1}{\Gamma(\alpha)} \frac{\partial}{\partial t} \int_{h(x)}^{t}(t-\tau)^{\alpha-1} \frac{\partial u}{\partial x}(x, \tau) \mathrm{d} \tau .
$$


Putting

$$
\mu_{\alpha}=\frac{1}{\nu_{\alpha}}
$$

from (16) and (13) it results that

$$
\left[\mu_{\alpha}\right]=\mathbf{t}^{1-\alpha}
$$

and

$$
\lim _{\alpha \nearrow 1} \mu_{\alpha}=1 .
$$

Then, equation (20) becomes

$$
J(x, t)=-k \mu_{\alpha} \underset{h(x)}{R L} D_{t}^{1-\alpha} \frac{\partial u}{\partial x}(x, t) .
$$

Remark 4. Fractional explicit expressions for the flux such as the given in (23) were considered in many publications (see for instance [30, 35]). Although it is a direct consequence of the formulation (12), up to now, the physical meaning of the partial derivative with respect to time in (23) was not clear.

Let us derive the governing equations of our problem. Note that the starting time being a function of $x$ in the fractional derivative, the governing equation will not coincide exactly with the subdiffusion equation given in Table 1.

Let $0<x<s(t), t>0$ be. Differentiating equation (12) respect to $x$ yields that

$$
\frac{\partial}{\partial x}\left(\nu_{\alpha h(x)} I_{t}^{1-\alpha} J(x, t)\right)=-k \frac{\partial^{2} u}{\partial x^{2}}(x, t) .
$$

Or equivalently,

$$
\frac{\partial}{\partial x}\left[\frac{\nu_{\alpha}}{\Gamma(1-\alpha)} \int_{h(x)}^{t}(t-\tau)^{-\alpha} J(x, \tau) d \tau\right]=-k \frac{\partial^{2} u}{\partial x^{2}}(x, t) .
$$

Differentiating the left-hand side of latter equation and using the continuity equation (2) we have

$$
\frac{\nu_{\alpha}}{\Gamma(1-\alpha)} \int_{h(x)}^{t}(t-\tau)^{-\alpha} \rho c \frac{\partial}{\partial t} u(x, \tau) d \tau+\nu_{\alpha} \lim _{\tau \searrow h(x)} \frac{(t-\tau)^{-\alpha}}{\Gamma(1-\alpha)} J(x, \tau) h^{\prime}(x)=k \frac{\partial^{2} u}{\partial x^{2}}(x, t) .
$$

Then the governing equation is

$$
\rho c_{h(x)}^{C} D_{t}^{\alpha} u(x, t)+\lim _{\tau \searrow h(x)} \frac{(t-\tau)^{-\alpha}}{\Gamma(1-\alpha)} J(x, \tau) h^{\prime}(x)=\frac{k}{\nu_{\alpha}} \frac{\partial^{2} u}{\partial x^{2}}(x, t) .
$$


Remark 5. In case we use the alternative flux definition (18), which is equivalent to (12), in the derivation steps of the governing equation, we get

$$
\frac{\partial}{\partial x}\left[\frac{\nu_{\alpha}}{\Gamma(1-\alpha)} \int_{0}^{t}(t-\tau)^{-\alpha} J(x, \tau) d \tau\right]=-k \frac{\partial^{2} u}{\partial x^{2}}(x, t) .
$$

It must be pointed out that the flux $J$ is not differentiable at $\tau=h(x) \in(0, t)$. Then, we can not differentiate under integral in the left-hand side of the latter equation. This fact is the main reason why we will not arrive to a single Caputo derivative over $[0, t]$ for $u$ in the left-hand side of equation (25), as has already been suggested in literature.

Now, we turn to study the moving interface. The interface is a curve where a discontinuity of the flux occurs. So, the energy balance between the latent heat and the difference of fluxes is given by the Rankine-Hugoniot conditions at the interface

$$
\llbracket \mathbf{J} \rrbracket_{l}^{s}=-\rho l \dot{s}(t) .
$$

Here, the double brackets represents the difference between the limits of the fluxes from the solid phase and the liquid phase. Recall that the explicit flux is given by (23) in the liquid phase, and the temperature is constant in the solid phase (which implies that the flux is null in this region as we have seen in Remark 3). Then condition (26) becomes

$$
\lim _{x \nearrow s(t)} J(x, t)=\rho l s^{\prime}(t)
$$

or equivalently (by using (23) )

$$
-k \mu_{\alpha} \lim _{x \nearrow_{s}(t)} R L(x) D_{t}^{1-\alpha} \frac{\partial u}{\partial x}(x, t)=\rho l s^{\prime}(t) .
$$

Making an abuse of language, we will call equation (27) the "fractional Stefan condition". Assuming the continuity of the flux in the liquid region, the following equality holds

$$
\lim _{x \nearrow s(t)} J(x, t)=\lim _{t \searrow h(x)} J(x, t) .
$$

Combining (25) and (28) we get the following governing equation for the liquid phase

$$
\rho c_{h(x)}^{C} D_{t}^{\alpha} u(x, t)+\frac{\rho l}{\Gamma(1-\alpha)} \frac{s^{\prime}(h(x)) h^{\prime}(x)}{(t-h(x))^{\alpha}}=\frac{k}{\nu_{\alpha}} \frac{\partial^{2} u}{\partial x^{2}}(x, t) .
$$

Being $h$ the inverse function of $s$, it results that

$$
h^{\prime}(x)=\frac{1}{s^{\prime}\left(s^{-1}(t)\right)}=\frac{1}{s^{\prime}(h(x))} .
$$


Finally, using (30) in (29) leads to

$$
\rho c_{h(x)}^{C} D_{t}^{\alpha} u(x, t)+\rho l \frac{(t-h(x))^{-\alpha}}{\Gamma(1-\alpha)}=\frac{k}{\nu_{\alpha}} \frac{\partial^{2} u}{\partial x^{2}}(x, t) .
$$

If we consider the Stefan number defined by

$$
\text { Ste }=\frac{c\left(T_{0}-T_{m}\right)}{l}, \quad([\text { Ste }]=1)
$$

and we use it in (31), we get

$$
{ }_{h(x)}^{C} D_{t}^{\alpha} u(x, t)+\frac{\left(T_{0}-T_{m}\right)}{S t e} \frac{(t-h(x))^{-\alpha}}{\Gamma(1-\alpha)}=\mu_{\alpha} d \frac{\partial^{2} u}{\partial x^{2}}(x, t),
$$

where $d$ is the diffusion coefficient defined in (11) and $\mu_{\alpha}$ was given in (21).

Note 3. It is easy to check that

$$
\left[{ }_{h}^{C} D_{t}^{\alpha} u+\frac{\left(T_{0}-T_{m}\right)}{S t e} \frac{(t-h(x))^{-\alpha}}{\Gamma(1-\alpha)}\right]=\left[\mu_{\alpha} d \frac{\partial^{2} u}{\partial x^{2}}\right]=\frac{T}{t^{\alpha}} .
$$

Note 4. We would like to highlight the difference between the fractional Stefan condition obtained in (27) and the fractional Stefan condition considered in [33] which was given by

$$
\rho l_{0}^{C} D^{\alpha} s(t)=-k \frac{\partial u}{\partial x}(s(t), t)
$$

and was derived by replacing the classical derivative by the Caputo derivative in the classical Stefan condition.

Finally, using equations (27) and (33), and adding appropriate initial conditions, the system representing the physical problem proposed at the beginning of the current section is given by

(i) $\quad{ }_{h(x)}^{C} D_{t}^{\alpha} u(x, t)+\frac{\left(T_{0}-T_{m}\right)}{S t e} \frac{(t-h(x))^{-\alpha}}{\Gamma(1-\alpha)}=\mu_{\alpha} d \frac{\partial^{2} u}{\partial x^{2}}(x, t), \quad 0<x<s(t), 0<t<T$,

(ii) $s(0)=0$,

(iii) $u(0, t)=T_{0}$,

$0<t \leq T$

(iv) $u(s(t), t)=T_{m}$,

$0<t \leq T$

(v) $\quad \rho l s^{\prime}(t)=-\mu_{\alpha} k \lim _{x \nearrow s(t)} \frac{R L}{h(x)} D_{t}^{1-\alpha} \frac{\partial}{\partial x} u(x, t), \quad 0<t \leq T$,

where $h(x)=s^{-1}(x)$ for every $x>0$.

Definition 2. A pair $\{u, s\}$ is a solution of problem (34) if the following conditions are satisfied 
1. $u$ is continuous in the region $\mathcal{R}_{\mathcal{T}}=\{(x, t): 0 \leq x \leq s(t), 0<t \leq T\}$ and at the point $(0,0), u$ verifies that

$$
0 \leq \liminf _{(x, t) \rightarrow(0,0)} u(x, t) \leq \limsup _{(x, t) \rightarrow(0,0)} u(x, t)<+\infty .
$$

2. $u \in C\left(\mathcal{R}_{\mathcal{T}}\right) \cap C_{x}^{2}\left(\mathcal{R}_{\mathcal{T}}\right)$, such that $u \in W_{t}^{1}((h(x), T))$ where $W_{t}^{1}((h(x), T)):=\{f(x, \cdot): f \in$ $W^{1}(h(x), T)$ for every fixed $\left.x \in[0, s(T)]\right\}$.

3. $s \in C^{1}(0, T)$.

4. There exists $\left.\underset{h(x)}{R L} D_{t}^{1-\alpha} \frac{\partial}{\partial x} u(x, t)\right|_{\left(s(t)^{-}, t\right)}$ for all $t \in(0, T]$.

5. $u$ and $s$ satisfy (34).

\section{Integral condition}

It is interesting to note that, from the definition (12) for the flux and Proposition 1 - 3, it results that expression (12) is equivalent to expression (19) for the flux. Then, if we replace (19) in the continuity equation (2) we obtain the following governing equation, which is a fractional diffusion equation for the Riemann-Liouville derivative:

$$
\frac{\partial u}{\partial t}(x, t)=\mu_{\alpha} d \frac{\partial}{\partial x}\left(\begin{array}{c}
R L \\
h(x)
\end{array} D_{t}^{1-\alpha} \frac{\partial}{\partial x} u(x, t)\right), \quad 0<x<s(t), t>0 .
$$

Lemma 1. The following jumping formulas hold:

1. If $w(x, \cdot)$ and $w_{x}(x, \cdot) \in L^{1}(0, T)$ then

$$
h(x) I_{t}^{1-\alpha}\left[\frac{\partial}{\partial x} w(x, t)\right]-\frac{\partial}{\partial x}\left[h(x) I_{t}^{1-\alpha} w(x, t)\right]=\lim _{\tau \searrow h(x)} w(x, \tau) \frac{(t-\tau)^{-\alpha}}{\Gamma(1-\alpha)} h^{\prime}(x) .
$$

2. If $w(x, \cdot) \in A C^{1}(0, T)$ and ${ }_{h(x)}^{R L} D_{t}^{1-\alpha}\left[\frac{\partial}{\partial x} w(x, t)\right]$ is a continuous function then

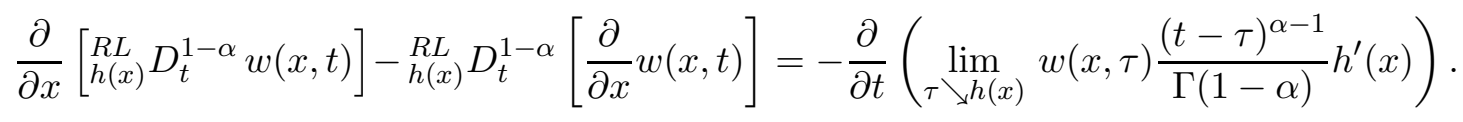

Proof. 1. Applying first the definition of fractional integral and differentiating with respect to $x$ we get

$$
\begin{array}{r}
\frac{\partial}{\partial x}\left[h(x) I_{t}^{1-\alpha} w(x, t)\right]=\frac{\partial}{\partial x}\left[\frac{1}{\Gamma(1-\alpha)} \int_{h(x)}^{t} w(x, \tau)(t-\tau)^{-\alpha} \mathrm{d} \tau\right] \\
=\frac{1}{\Gamma(1-\alpha)}\left[\int_{h(x)}^{t} \frac{\partial}{\partial x} w(x, \tau)(t-\tau)^{-\alpha} \mathrm{d} \tau-\lim _{\tau \searrow h(x)} w(x, \tau)(t-\tau)^{-\alpha} h^{\prime}(x)\right]
\end{array}
$$


Equation (35) can be derived directly from (36).

2. Analogously,

$$
\begin{aligned}
& \frac{\partial}{\partial x}\left[{ }_{h(x)}^{R L} D_{t}^{1-\alpha} w(x, t)\right]=\frac{\partial}{\partial x} \frac{\partial}{\partial t}\left[h(x) I_{t}^{\alpha} w(x, t)\right] \\
&=\frac{\partial}{\partial t} \frac{\partial}{\partial x}\left[{ }_{h(x)} I_{t}^{\alpha} w(x, t)\right]=\frac{\partial}{\partial t} \frac{\partial}{\partial x}\left[\frac{1}{\Gamma(\alpha)} \int_{h(x)}^{t} w(x, \tau)(t-\tau)^{\alpha-1} \mathrm{~d} \tau\right] \\
&={ }_{h(x)}^{R L} D_{t}^{1-\alpha}\left[\frac{\partial}{\partial x} w(x, t)\right]-\frac{\partial}{\partial t}\left(\lim _{\tau \searrow h(x)} \frac{w(x, \tau)(t-\tau)^{\alpha-1}}{\Gamma(\alpha)} h^{\prime}(x)\right) .
\end{aligned}
$$

Proposition 3. Consider the following fractional Stefan problem

(i) $\quad \frac{\partial u}{\partial t}(x, t)=\mu_{\alpha} d \frac{\partial}{\partial x}\left({ }_{h(x)}^{R L} D_{t}^{1-\alpha} \frac{\partial}{\partial x} u(x, t)\right), \quad 0<x<s(t), 0<t<T$,

(ii) $s(0)=0$,

(iii) $u(0, t)=T_{0}>T_{m}, \quad 0<t \leq T$,

(iv) $u(s(t), t)=T_{m}, \quad 0<t \leq T$,

(v) $\quad \rho l s^{\prime}(t)=-\mu_{\alpha} k \lim _{x \nearrow_{s(t)}} R L(x) D_{t}^{1-\alpha} \frac{\partial}{\partial x} u(x, t), \quad 0<t \leq T$.

where $h$ is the function defined by $h(x)=s^{-1}(x)$. Then problems (34) and (37) are equivalent.

Proof. Being equations $(i i)$ to $(v)$ the same in both problems we have to check only that equations $(34-i)$ and $(37-i)$ are equivalent.

Applying $_{h(x)} I_{t}^{1-\alpha}$ to both sides of $(37-i)$ we get

$$
{ }_{h(x)}^{C} D_{t}^{\alpha} u(x, t)=\mu_{\alpha} d_{h(x)} I_{t}^{1-\alpha}\left[\frac{\partial}{\partial x}\left({ }_{h(x)}^{R L} D_{t}^{1-\alpha} \frac{\partial}{\partial x} u(x, t)\right)\right] .
$$

Proposition 1-1 implies that if we apply $\underset{h(x)}{R L} D_{t}^{1-\alpha}$ to both sides of (38) we recover equation $(37-i)$. Therefore $(37-i)$ and (38) are equivalent.

On one hand, taking $w(x, t)={ }_{h(x)}^{R L} D_{t}^{1-\alpha}\left(\frac{\partial}{\partial x} u(x, t)\right)$ in Lemma 1, 1 we get

$$
\begin{aligned}
& \frac{\partial}{\partial x}\left(h(x) I_{t}^{1-\alpha} \underset{h(x)}{R L} D_{t}^{1-\alpha}\left(\frac{\partial}{\partial x} u(x, t)\right)\right)=\frac{\partial}{\partial x}\left[\frac{1}{\Gamma(1-\alpha)} \int_{h(x)}^{t}(t-\tau)^{-\alpha} \underset{h(x)}{R L} D_{t}^{1-\alpha}\left(\frac{\partial}{\partial x} u(x, \tau)\right) \mathrm{d} \tau\right] \\
= & { }_{h(x)} I_{t}^{1-\alpha}\left[\frac{\partial}{\partial x}\left({ }_{h(x)}^{R L} D_{t}^{1-\alpha}\left(\frac{\partial}{\partial x} u(x, t)\right)\right)\right]-\lim _{\tau \searrow h(x)} \underset{h L}{R L} D_{t}^{1-\alpha}\left(\frac{\partial}{\partial x} u(x, t)\right) \frac{(t-\tau)^{-\alpha}}{\Gamma(1-\alpha)} h^{\prime}(x) .
\end{aligned}
$$


On the other hand, from (12) and Proposition 1 - 3 , it holds that

$$
h(x) I_{t}^{1-\alpha}\left(\underset{h L(x)}{R L} D_{t}^{1-\alpha}\left(\frac{\partial}{\partial x} u(x, t)\right)\right)=\frac{\partial}{\partial x} u(x, t) .
$$

Then (39) together with (40) yield

$$
\begin{aligned}
\mu_{\alpha} d_{h(x)} I_{t}^{1-\alpha}\left[\frac{\partial}{\partial x}\left({ }_{h(x)}^{R L} D_{t}^{1-\alpha}\left(\frac{\partial}{\partial x} u(x, t)\right)\right)\right] & = \\
& =\mu_{\alpha} d \frac{\partial^{2}}{\partial x^{2}} u(x, t)-\frac{d}{\Gamma(1-\alpha)} \frac{\rho l}{k} \frac{s^{\prime}(h(x)) h^{\prime}(x)}{(t-h(x))^{\alpha}} .
\end{aligned}
$$

So, we can rewrite equation (38) as

$$
{ }_{h(x)}^{C} D_{t}^{\alpha} u(x, t)=\mu_{\alpha} d \frac{\partial^{2}}{\partial x^{2}} u(x, t)-\frac{d}{\Gamma(1-\alpha)} \frac{\rho l}{k} \frac{s^{\prime}(h(x)) h^{\prime}(x)}{(t-h(x))^{\alpha}} .
$$

Taking into account (30) and (32), we conclude that (41) is equivalent to (34-i), and then the thesis holds.

Definition 3. A pair $\{u, s\}$ is a solution of problem (37) if the following conditions are satisfied

1. $u$ is continuous in the region $\mathcal{R}_{\mathcal{T}}=\{(x, t): 0 \leq x \leq s(t), 0<t \leq T\}$ and at the point $(0,0), u$ verifies that

$$
0 \leq \liminf _{(x, t) \rightarrow(0,0)} u(x, t) \leq \limsup _{(x, t) \rightarrow(0,0)} u(x, t)<+\infty .
$$

2. $u \in C\left(\mathcal{R}_{\mathcal{T}^{\circ}}\right) \cap C_{x}^{2}\left(\mathcal{R}_{\mathcal{T}^{\circ}}\right)$, such that $u_{x} \in A C_{t}^{1}((h(x), T))$ where $A C_{t}^{1}((h(x), T)):=$ $\left\{f(x, \cdot): f \in A C^{1}(h(x), T)\right.$ for every fixed $\left.x \in[0, s(T)]\right\}$.

3. $s \in C^{1}(0, T)$.

4. There exists $\left.{ }_{0}^{R L} D_{t}^{1-\alpha} \frac{\partial}{\partial x} u(x, t)\right|_{(s(t), t)}$ for all $t \in(0, T]$.

5. $u$ and $s$ satisfy (37).

Lemma 2. If the pair $\{u, s\}$ is a solution to problem (37) and $\frac{\partial}{\partial x}\left[\begin{array}{l}R L \\ h(x)\end{array} D_{t}^{1-\alpha} u(x, t)\right]$ is a continuous function, then

$$
\frac{\partial}{\partial x} \underset{h(x)}{C} D_{t}^{1-\alpha} u(x, t)={ }_{h(x)}^{R L} D_{t}^{1-\alpha}\left(\frac{\partial}{\partial x} u(x, t)\right) .
$$


Proof. Since $\frac{\partial}{\partial x}\left[{ }_{h(x)}^{R L} D_{t}^{1-\alpha} u(x, t)\right]$ is a continuous function, the partial derivatives commutes and

$$
\begin{aligned}
\frac{\partial}{\partial x}\left[\begin{array}{l}
R L \\
h(x)
\end{array} D_{t}^{1-\alpha} u(x, t)\right] & =\frac{\partial}{\partial t} \frac{1}{\Gamma(\alpha)} \frac{\partial}{\partial x} \int_{h(x)}^{t} u(x, \tau)(t-\tau)^{\alpha-1} d \tau \\
& =\frac{\partial}{\partial t} \frac{1}{\Gamma(\alpha)}\left[\int_{h(x)}^{t}\left(\frac{\partial}{\partial x} u(x, \tau)\right)(t-\tau)^{\alpha-1} d \tau-u(x, h(x))(t-h(x))^{\alpha-1} h^{\prime}(x)\right] \\
& ={ }_{h(x)}^{R L} D_{t}^{1-\alpha}\left(\frac{\partial}{\partial x} u(x, t)\right)-\frac{\partial}{\partial t} \frac{T_{m}}{\Gamma(\alpha)}(t-h(x))^{\alpha-1} h^{\prime}(x) \\
& ={ }_{h(x)}^{R L} D_{t}^{1-\alpha}\left(\frac{\partial}{\partial x} u(x, t)\right)+\frac{T_{m}(1-\alpha)}{\Gamma(\alpha)} \frac{h^{\prime}(x)}{(t-h(x))^{2-\alpha}} \\
& ={ }_{h(x)}^{R L} D_{t}^{1-\alpha}\left(\frac{\partial}{\partial x} u(x, t)\right)+\frac{\partial}{\partial x}\left[\frac{T_{m}}{\Gamma(\alpha)(t-h(x))^{1-\alpha}}\right]
\end{aligned}
$$

Then

$$
\frac{\partial}{\partial x}\left[\underset{h(x)}{R L} D_{t}^{1-\alpha} u(x, t)-\frac{T_{m}}{\Gamma(\alpha)(t-h(x))^{1-\alpha}}\right]=\underset{h(x)}{R L} D_{t}^{1-\alpha}\left(\frac{\partial}{\partial x} u(x, t)\right) .
$$

Applying Proposition 1 14 in (42) leads to

$$
\frac{\partial}{\partial x} \underset{h(x)}{C} D_{t}^{1-\alpha} u(x, t)={ }_{h(x)}^{R L} D_{t}^{1-\alpha}\left(\frac{\partial}{\partial x} u(x, t)\right) .
$$

This concludes the proof.

Theorem 1. Let $\{u, s\}$ be a solution of problem (37) with $u$ such that ${ }_{h(x)}^{R L} D_{t}^{1-\alpha} u(x, t)$ and ${ }_{h(x)}^{R L} D_{t}^{1-\alpha}\left(\frac{\partial}{\partial x} u(x, t)\right)$ are in $\mathcal{C}^{1}\left(\mathcal{R}_{T}-\{(0,0)\}\right)$. Then the following integral relation for the free boundary $s(t)$ and the function $u(x, t)$ holds for every $t<T$ :

$$
\left(\frac{l}{c}-T_{m}\right) s^{2}(t)=2 \mu_{\alpha} d \frac{T_{0}-T_{m}}{\Gamma(\alpha+1)} t^{\alpha}-2 \int_{0}^{s(t)} x u(x, t) d x-\left.2 \mu_{\alpha} d \int_{0}^{t} \underset{h(x)}{C} D_{t}^{1-\alpha} u(x, t)\right|_{(s(\tau), \tau)} d \tau .
$$

Proof. Recall the Green identity:

$$
\int_{\partial \Omega} P d t+Q d x=\iint_{\Omega}\left(\frac{\partial}{\partial t} Q-\frac{\partial}{\partial x} P\right) d A
$$


where $\Omega$ is an open simply connected region, $\partial \Omega$ is a positively oriented, piecewise smooth, simple closed curve, and the field $F=(P, Q)$ is defined by

$$
\begin{gathered}
P(x, t)=-\mu_{\alpha} d x_{h(x)}^{R L} D_{t}^{1-\alpha}\left(\frac{\partial}{\partial x} u(x, t)\right)+\mu_{\alpha} d_{h(x)}^{R L} D_{t}^{1-\alpha}\left(u(x, t)-T_{m}\right) \\
=-\mu_{\alpha} d x_{h(x)}^{R L} D_{t}^{1-\alpha}\left(\frac{\partial}{\partial x} u(x, t)\right)+\mu_{\alpha} d_{h(x)}^{C} D_{t}^{1-\alpha} u(x, t) \\
Q(x, t)=-x u(x, t) .
\end{gathered}
$$

Consider the region $\mathcal{R}_{t}{ }^{\epsilon}=\left\{(x, \tau) \in \mathbb{R}^{2} / \epsilon<\tau<t, 0<x<s(\tau)\right\}$ for $\epsilon>0$ sufficiently small. Note that in this region, $F$ is $\mathcal{C}^{1}$. Now, taking into account that $u$ verifies $(37-i)$ and using Lemma 2 we get

$$
\begin{aligned}
& \frac{\partial}{\partial t} Q(x, t)-\frac{\partial}{\partial x} P(x, t)= \\
& =-x \frac{\partial}{\partial t} u(x, t)+\mu_{\alpha} d_{h(x)}^{R L} D_{t}^{1-\alpha}\left(\frac{\partial}{\partial x} u(x, t)\right) \\
& \quad+\mu_{\alpha} d x \frac{\partial}{\partial x}\left[{ }_{h(x)}^{R L} D_{t}^{1-\alpha}\left(\frac{\partial}{\partial x} u(x, t)\right)\right]-\mu_{\alpha} d \frac{\partial}{\partial x}\left[{ }_{h(x)}^{C} D_{t}^{1-\alpha} u(x, t)\right] \\
& =-x\left[\frac{\partial}{\partial t} u(x, t)-\mu_{\alpha} d \frac{\partial}{\partial x}\left({ }_{h(x)}^{R L} D_{t}^{1-\alpha}\left(\frac{\partial}{\partial x} u(x, t)\right)\right)\right] \\
& \quad+\mu_{\alpha} d{ }_{h(x)}^{R L} D_{t}^{1-\alpha}\left(\frac{\partial}{\partial x} u(x, t)\right)-\mu_{\alpha} d \frac{\partial}{\partial x}\left[{ }_{h(x)}^{C} D_{t}^{1-\alpha} u(x, t)\right] \\
& =0 \quad \text { for all }(x, t) \in \mathcal{R}_{\epsilon} .
\end{aligned}
$$

Then, by Green's theorem one obtains

$$
\int_{\partial \mathcal{R}_{\epsilon}} P d \tau+Q d x=0
$$

Let $\partial \mathcal{R}_{\epsilon}=\partial \mathcal{R}_{1} \cup \partial \mathcal{R}_{2} \cup \partial \mathcal{R}_{3} \cup \partial \mathcal{R}_{4}$ be, where $\partial \mathcal{R}_{1}=\{(x, \epsilon): 0 \leq x \leq s(\epsilon)\}$, $\partial \mathcal{R}_{2}=\{(s(\tau), \tau): \epsilon<\tau<t\},-\partial \mathcal{R}_{3}=\{(x, t): 0 \leq x \leq s(t)\}$ and $-\partial \mathcal{R}_{4}=\{(0, \tau): \epsilon \leq \tau \leq t\}$.

Integrating the field $(P, Q)$ over $\partial \mathcal{R}_{\epsilon}$ we get

$$
\begin{aligned}
& -\int_{0}^{s(\epsilon)} x u(x, \epsilon) \mathrm{d} x-\int_{\epsilon}^{t}\left[\left.\mu_{\alpha} d_{h(x)}^{R L} D_{t}^{1-\alpha}\left(\frac{\partial}{\partial x} u(x, t)\right)\right|_{(s(\tau), \tau)}\right. \\
& \left.+\left.\mu_{\alpha} d_{h(x)}^{C} D_{t}^{1-\alpha} u(x, t)\right|_{(s(\tau), \tau)}\right] \mathrm{d} \tau- \\
& -\int_{\epsilon}^{t} s(\tau) T_{m} s^{\prime}(\tau) \mathrm{d} \tau+\int_{0}^{s(t)} x u(x, t) \mathrm{d} x-\int_{\epsilon}^{t} \mu_{\alpha} d_{h(x)}^{C} D_{t}^{1-\alpha} T_{0} \mathrm{~d} \tau=0 .
\end{aligned}
$$


Applying the fractional Stefan condition (37) yields

$$
\begin{aligned}
-\int_{0}^{s(\epsilon)} x u(x, \epsilon) d x+\left(\frac{l}{c}-T_{m}\right) & {\left[\frac{s(t)^{2}}{2}-\frac{s(\epsilon)^{2}}{2}\right]+\left.\mu_{\alpha} d \int_{\epsilon}^{t}{ }_{h(x)}^{C} D_{t}^{1-\alpha} u(x, t)\right|_{(s(\tau), \tau)} } \\
& +\int_{0}^{s(t)} x u(x, t) d x-\mu_{\alpha} d \frac{\left(T_{0}-T_{m}\right)}{\Gamma(\alpha+1)}\left(t^{\alpha}-\epsilon^{\alpha}\right)=0 .
\end{aligned}
$$

Taking the limit when $\epsilon \searrow 0$ in (45) it results that the integral relation (43) holds as we wanted to prove.

Remark 6. It is worth noting the difference between

$$
\left.{ }_{h(x)}^{C} D_{t}^{1-\alpha} u(x, t)\right|_{(s(\tau), \tau)},
$$

and

$$
{ }_{h(x)}^{C} D_{t}^{1-\alpha} u(s(t), t)={ }_{h(x)}^{C} D_{t}^{1-\alpha} T_{m}=0 .
$$

In (46) we first apply Caputo derivative and then evaluate at $(s(t), t)$. Instead, in (47) we first evaluate function $u$ in $(s(t), t)$ and then the Caputo derivative is taken.

Remark 7. If we take $\alpha=1, T_{m}=0$ and all the physical constants equal to 1 in the integral relation (43) we get

$$
s^{2}(t)=-2 \int_{0}^{s(t)} x u(x, t) d x+2 T_{0} t
$$

which is the classical integral relation for the free boundary when the classical Stefan problem is considered (see [5]-Lemma 17.1.1).

It was also proved in [5] that (17) is equivalent to the Stefan condition

$$
s^{\prime}(t)=-\frac{\partial}{\partial x} u(s(t), t), \quad \forall t>0 .
$$

Hence, it is natural to wonder if the "fractional Stefan condition" (27) and the "fractional integral relation" (43) are equivalent as well.

Theorem 2. Let $\{u, s\}$ be a solution of problem $\{(37-i),(37-i i),(37-i i i),(37-i v),(43)\}$ such that $\frac{\partial^{2}}{\partial t \partial x} u(x, t) \in \mathcal{C}^{1}\left(\mathcal{R}_{T}\right),\left.{ }_{h(x)}^{C} D_{t}^{1-\alpha} u(x, t)\right|_{(s(t), t)} \in L^{1}(0, T)$. Then the functions $s=s(t)$ and $u=u(x, t)$ verify the fractional Stefan condition (27).

Proof. Reasoning as in Theorem 1, we can state that again (44) holds.

Taking the limit when $\epsilon \searrow 0$ and using the integral relation (43) it holds that

$$
\frac{l}{c} s^{2}(t)=-\left.2 \mu_{\alpha} d \int_{0}^{t} s(\tau)^{R L} D_{t}^{1-\alpha} \frac{\partial}{\partial x} u(x, t)\right|_{(s(\tau), \tau)} d \tau .
$$

Differentiating both sides of equation (49) whith respect to the $t$-variable and being $s(t)>0$ for all $t>0$, the thesis holds. 


\section{Conclusions}

We have presented a physical phase change problem involving a material with memory. In the mathematical model, a fractional Riemann-Liouville integral is used for an implicit definition of the flux. Then, the governing equations were obtained. As a result of this analysis two equivalent fractional Stefan problems (34) and (37) involving the Caputo and the Riemann-Liouville derivative, respectively, were formulated. The comparison with the classical Stefan problem was given in each case. Moreover, the classical Stefan problem was recovered by making $\alpha \nearrow 1$. Finally, an integral relation which is equivalent to the fractional Stefan condition was obtained.

\section{Acknowledgements}

The present work has been sponsored by the Projects PIP $\mathrm{N}^{\circ} 0275$ from CONICETUniv. Austral, and ANPCyT PICTO Austral N0090 (Rosario, Argentina). We appreciate the valuable suggestions by the anonymous referees which helped us to improve the paper. We are particularly grateful for the assistance given by Prof. María Soledad Aronna.

\section{References}

[1] M. Al-Refai and Y. Luchko. Maximum principle for the fractional diffusion equations with the Riemann-Liouville fractional derivative and its applications. Fractional Calculus \& Applied Analysis, 17(2):483-498, 2014.

[2] C. Atkinson. Moving boundary problems for time fractional and composition dependent diffusion. Fractional Calculus \& Applied Analysis, 15(2):207-221, 2012.

[3] Daniel S. Banks and Cécile Fradin. Anomalous diffusion of proteins due to molecular crowding. Biophysical Journal, 89(5):2960 - 2971, 2005.

[4] M. Miksis C. Gruber, C. Vogl and S. Davis. Anomalous diffusion models in the presence of a moving interface. Interfaces and Free Boundaries, 15:181-202, 2013.

[5] J. R. Cannon. The One-Dimensional Heat Equation. Cambridge University Press, 1984.

[6] C. Cattaneo. Sulla conduzione del calore. Atti del Seminario Matematico e Físico dell'Università di Modena , 3:83-101, 1948.

[7] A.N. Ceretani. A note on Stefan-like models for phase-change processes in nonhomogeneous media. Available in https://arxiv.org/abs/1801.10069.pdf, 2018. 
[8] D. S. Chandrasekharaiah. Hyperbolic thermoelasticity: a review of recent literature. Applied Mechanics Reviews, 51:705-729, 1986.

[9] K. Diethelm. The Analysis of Fractional Differential Equations: An application oriented exposition using differential operators of Caputo type, volume 2004. Springer, 2010 .

[10] S. D. Eidelman, S. D. Ivasyshen, and A. N. Kochubei. Analytic Methods in the Theory of Differential and Pseudo-Differential Equations of Parabolic Type. Birkhäuser Verlag, 2004 .

[11] S. D. Eidelman and A. N. Kochubei. Cauchy problem for fractional diffusion equations. Journal of Differential Equations, 199:211-255, 2004.

[12] D. N. Gerasimov, V. A. Kondratieva, and O. A. Sinkevich. An anomalous non-selfsimilar infiltration and fractional diffusion equation. Physica D: Nonlinear Phenomena, 239(16):1593-1597, 2010.

[13] D. Goos, G. Reyero, S. Roscani, and E. Santillan Marcus. On the initial-boundaryvalue problem for the time-fractional diffusion equation on the real positive semiaxis. International Journal of Differential Equations, Article ID 439419:1-14, 2015.

[14] R. Gorenflo, Y. Luchko, and F. Mainardi. Wright functions as scale-invariant solutions of the diffusion-wave equation. Journal of Computational and Applied Mathematics, 118(1):175-191, 2000.

[15] M. E. Gurtin and A. C. Pipkin. A general theory of heat conduction with finite wave speeds. Archive for Rational Mechanics and Analysis, 31(2):113-126, 1968.

[16] R.B. Hetnarski and J. Ignaczak. Generalized thermoelasticity. Journal of Thermal Stresses, 22:451-476, 1999.

[17] Ignaczak J. and Ostoja-Starzewski M. Thermoelasticity with Finite Wave Speeds. University Press, 2010.

[18] D. D. Joseph and L. Preziosi. Heat waves. Reviews of Moderns Physics, 61:41-73, 1989.

[19] J. Klafter and I. Sokolov. Anomalous diffusion spreads its wings. Physics Word, 18(8):29, 2005.

[20] X. Li, M. Xu, and X. Jiang. Homotopy perturbation method to time-fractional diffusion equation with a moving boundary condition. Applied Mathematics and Computation, 208:434-439, 2009. 
[21] Y. Liu. Strong maximum principle for multi-term time-fractional diffusion equations and its application to an inverse source problem. Computers and Mathematics with Applications, 73(1):96 - 108, 2017.

[22] Y. Luchko. Maximum principle for the generalized time-fractional diffusion equation. Journal of Mathematical Analysis and Applications, 351:218-223, 2009.

[23] Y. Luchko, F. Mainardi, and G. Pagnini. The fundamental solution of the space-time fractional diffusion equation. Fractional Calculus \& Applied Analysis, 4(2):153-192, 2001.

[24] F. Mainardi. Fractional Calculus and Waves in Linear Viscoelasticity. Imperial Collage Press, 2010.

[25] R. Metzler, W. Gloeckle, and T. Nonnenmacher. Fractional model equation for anomalous diffusion. Physica A, 211:13-24, 1994.

[26] R. Metzler and J. Klafter. The random walk's guide to anomalous diffusion: a fractional dynamics approach. Physics reports, 339:1-77, 2000.

[27] R. Nigmatullin. To the theoretical explanation of the "universal response". physica status solidi (b), 123(2):739-745, 1984.

[28] G. Pagnini. The M-Wright function as a generalization of the gaussian density for fractional diffusion processes. Fractional Calculus and Applied Analysis, 16(2):436453, 2013.

[29] Y. Povstenko. Signaling problem for time-fractional diffusion equation in a half-plane. Fractional Calculus \& Applied Analysis, 11(3):329-352, 2008.

[30] Y. Povstenko. Linear Fractional Diffusion-wave Equation for Scientists and Engineers. Springer, 2015.

[31] A. V. Pskhu. Partial Differential Equations of Fractional Order (in Russian). Nauka, Moscow, 2005.

[32] S. Roscani. Hopf lemma for the fractional diffusion operator and its application to a fractional free-boundary problem. Journal of Mathematical Analysis and Applications, 434:125-135, 2016.

[33] S. Roscani and E. Santillan Marcus. Two equivalen Stefan's problems for the timefractional diffusion equation. Fractional Calculus \& Applied Analysis, 16(4):802-815, 2013. 
[34] S. Roscani and D. Tarzia. A generalized Neumann solution for the two-phase fractional Lamé-Clapeyron-Stefan problem. Advances in Mathematical Sciences and Applications, 24(2):237-249, 2014.

[35] S. Roscani and D. Tarzia. An integral relationship for a fractional one-phase Stefan problem. Fractional Calculus \& Applied Analysis, In press(Available in https://arxiv.org/abs/1609.05169.pdf), 2018.

[36] S. Roscani and D. Tarzia. Two different fractional Stefan problems which are convergent to the same classical Stefan problem. Mathematical Methods in the Applied Sciences, In press(Available in https://arxiv.org/abs/1710.07620), 2018.

[37] Podstrigach Y. S. and Kolyano Y. M. Generalized Thermomechanics. Naukova Dumka (in russian), 1976.

[38] K. Sakamoto and M. Yamamoto. Initial value/boundary value problems for fractional diffusion-wave equations and applications to some inverse problems. Journal of Mathematical Analysis and Applications, 382:426-447, 2011.

[39] S. G. Samko, A. A. Kilbas, and O. I. Marichev. Fractional Integrals and DerivativesTheory and Applications. Gordon and Breach, 1993.

[40] M.J. Saxton. Anomalous diffusion due to obstacles: a monte carlo study. Biophysical Journal, 66(2, Part 1):394 - 401, 1994.

[41] D. A. Tarzia. A bibliography on moving-free boundary problems for the heat diffusion equation. the Stefan and related problems. MAT-Serie A, 2:1-297, 2000.

[42] D. A. Tarzia. Explicit and Approximated Solutions for Heat and Mass Transfer Problems with a Moving Interface, chapter 20, Advanced Topics in Mass Transfer, pages 439-484. Prof. Mohamed El-Amin (Ed.), Intech, Rijeka, 2011.

[43] C. J. Vogl, M. J. Miksis, and S. H. Davis. Moving boundary problems governed by anomalous diffusion. Proceedings of the Royal Society A, 468:3348-3369, 2012.

[44] V. R. Voller. An exact solution of a limit case Stefan problem governed by a fractional diffusion equation. International Journal of Heat and Mass Transfer, 53(23-24):5622$5625,2010$.

[45] V. R. Voller. Fractional Stefan problems. International Journal of Heat and Mass Transfer, 74:269-277, 2014.

[46] V. R. Voller, F. Falcini, and R. Garra. Fractional Stefan problems exhibing lumped and distributed latent-heat memory effects. Physical Review E, 87:042401, 2013. 